\title{
Radiation Damage to Scintillator in the DØ Luminosity Monitor
}

\author{
Brendan Casey, Kayle DeVaughan, Yuji Enari, Richard Partridge, and Sahal Yacoob
}

\begin{abstract}
We report the result of evaluating radiation damage to Bicron BC408 plastic scintillator used in the DØ Luminosity Monitor during Run IIa. The Luminosity Monitor provides pseudo-rapidity coverage over the range $2.7<|\eta|<4.4$, with the radiation dose in Run IIa estimated to be 0.5 MRad for the region closest to the beams. We find the light yield is degraded by $10-15 \%$ due to radiation damage by comparing new and old scintillator in four observables: (i) visual inspection, (ii)optical transmittance, (iii) response to the radioactive source of ${ }^{90} \mathrm{Sr}$ and (iv) light yield for cosmic rays.
\end{abstract}

Index Terms-Scintillator, Radiation damage, TeVatron, Luminosity.

\section{INTRODUCTION}

$\mathbf{T}$ HE DØ Luminosity Monitor (LM) consists of two array of scintillation counters located on the inside face of the endcap calorimeters, $140 \mathrm{~cm}$ from the center of the $\mathrm{D} \emptyset$ detector along the $\mathrm{z}$ direction (beam axis), and arranged symmetrically about the beam pipe. The detector covers a region in pseudo rapidity of $2.7<|\eta|<4.4$, providing an acceptance of 98 $\%$ for detecting non-diffractive inelastic collisions. Each of the LM arrays consists of 24 identical $1.6 \mathrm{~cm}$ thick BC-408 scintillator wedges, with Hamamatsu 1 inch diameter finemesh photo-multiplier tubes (R7474) mounted directly on the faces at $73.0 \mathrm{~mm}$ from narrow edge to center of PMT using silicone glue (GE RTV615). During 2001 March to 2006 February (RunIIa), the TeVatron accelerator complex delivered an integrated luminosity of $\sim 1.5 \mathrm{fb}^{-1}$, leading to an estimated radiation dose of $0.5 \mathrm{MRad}$ for the region closest to the beams. An investigation of the resulting scintillator radiation damage was performed during TeVatron shutdown (2006 March to 2006 June).

\section{STUdy OF RADiATION DAMAGE}

There are two possible sources of radiation damage to the scintillator. One is degradation in the scintillation mechanism itself. Bross and Pla-Dalmau have previously studied radiation damage in the scintillation mechanism for plastic scintillators [1]. They observed light loss from the scintillator mechanism after $10 \mathrm{MRad}$ irradiation due to absorption in the 'Hidden sector', which is the wavelength region below 400

Brendan Casey, Yuji Enari, Richard Partridge are with Department of Physics, Brown University, RI, USA

Kayle DeVaughan is with Department of Physics, University of Nebraska Lincoln, NE, USA

Sahal Yacoob is with Department of Physics \& Astronomy, Northwestern University, IL, USA

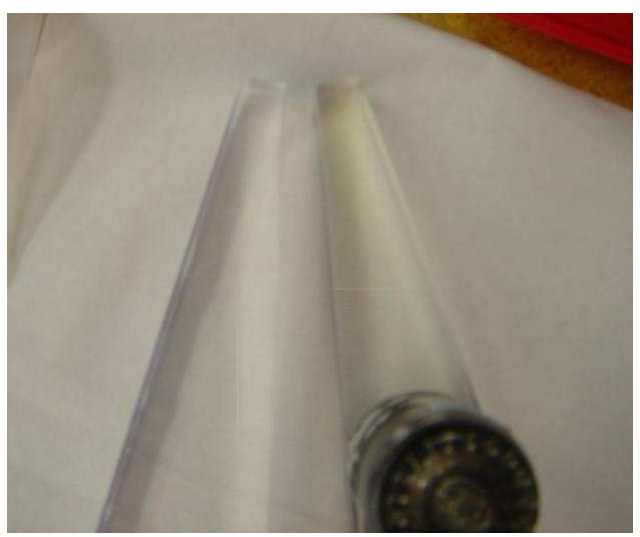

Fig. 1. Photograph of new (left) and exposed (right) scintillator. The narrow edge of exposed scintillator has a slight yellow coloration.

nm. Scintillation light produced directly by ionizing radiation is converted to longer wavelength by fluorescence in the wavelength shifter dopant. Absorption in the region from $330 \mathrm{~nm}$ to $380 \mathrm{~nm}$ attenuates the direct scintillation light, causing the output light from wave length shifter to also be reduced.

The other source of radiation damage is absorption of the wavelength shifted light due to degradation in the transmittance of the scintillator substrate. We expect that absorption of the wavelength shifted light is the main contribution to radiation damage in the LM scintillator since the estimated radiation dose is $0.5 \mathrm{MRad}$ where the effect of radiation damage to the scintillation mechanism is small.

In order to evaluate the radiation damage, we take two approaches. One is to measure the optical transmittance of the scintillator, and the other is to measure the light response from charged particle irradiation. The first approach provides proof that radiation damage has resulted in a loss of light transmittance in the scintillator substrate, and employs visual inspection and measuring the light transmittance using a Spectrophotometer. The second approach evaluates the detected light output yield. All 48 scintillator wedges in the LM were measured using a radioactive $\beta$ source. In addition, the light yield distribution as function of position in the scintillator was measured using cosmic rays for three scintillator wedges. By comparing this result with the transmittance measurement, we can check for consistency with our expectation that absorption in the scintillator substrate is the dominant source of radiation damage. 


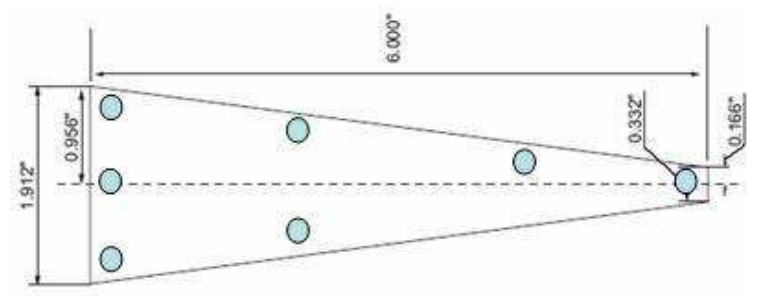

Fig. 2. The Position of light axis in transmittance measurement. Position 1 is centered on the narrow edge closest to the beams and positions 2-7 are follow a counter-clockwise ordering around the perimeter of the scintillator. The light spot is less than $1 \mathrm{~mm}$ in diameter.

\section{VISUAL INSPECTION}

We observed clear indication of radiation damage through visual inspection of the scintillator, as shown in Fig. 1. Compared to the new scintillator, the exposed scintillator is less transparent and has a noticeable yellow coloration in the narrow region that is closest to the beam axis.

\section{OPTICAL TRANSMITTANCE OF SCINTILLATOR}

In order to evaluate the optical transmittance of the scintillator, we used an HP 8452A spectrophotometer. The HP8452A has a well calibrated white light source that is chromatically dispersed onto a photo diode array, allowing simultaneous transmittance measurement over the wavelength range $190 \mathrm{~nm}$ to $820 \mathrm{~nm}$. The scintillator is set perpendicular to light axis so the light passes through the $1.6 \mathrm{~cm}$ scintillator thickness. By taking the ratio of the light yield with and without scintillator (air), we obtain the transmittance of the scintillator. We measured the transmittance for two irradiated and one new scintillator at seven points in each scintillator. The position of light axis on the scintilltor is shown in Fig. 2. By using an aluminum holder for positioning the scintillator, the position and angle of the light axis through the scintillator are controlled within $0.1 \mathrm{~mm}$ and $1 \mathrm{mrad}$, respectively.

The transmittance of the new scintillator is shown in Fig. 3. We observe a sharp turn on due to strong absorption below 407 $\mathrm{nm}$ due to absorption by the wavelength shifter, and smaller absorption dips at approximately $500 \mathrm{~nm}, 570 \mathrm{~nm}$, and 650 $\mathrm{nm}$ in all positions. Additionally we notice the transmittance is affected by light scattering on the surface scratches as well as bulk attenuation. We attribute the variation in transmittance above $500 \mathrm{~nm}$ to surface effects, which fluctuate from one position to another. To estimate the absorption due to radiation damage, we need to first account for the wavelength shifter absorption and the surface effects. We do this by fitting the measured transmittance $T_{\text {meas }}$ to the following empirical form

$$
T_{f i t}=\frac{T_{0}-K / \lambda}{1+\exp \left(-\left(\lambda-\lambda_{0}\right) / \Delta \lambda\right)},
$$

where $T_{0}$ and $K$ parameterize the surface effects and are free parameters in the fitting procedure, and $\lambda_{0}=407.7 \mathrm{~nm}$ and $\Delta \lambda=3.2 \mathrm{~nm}$ parameterize the wave length shifter absorption and are fixed in the fitting. We expect that irradiated scintillator
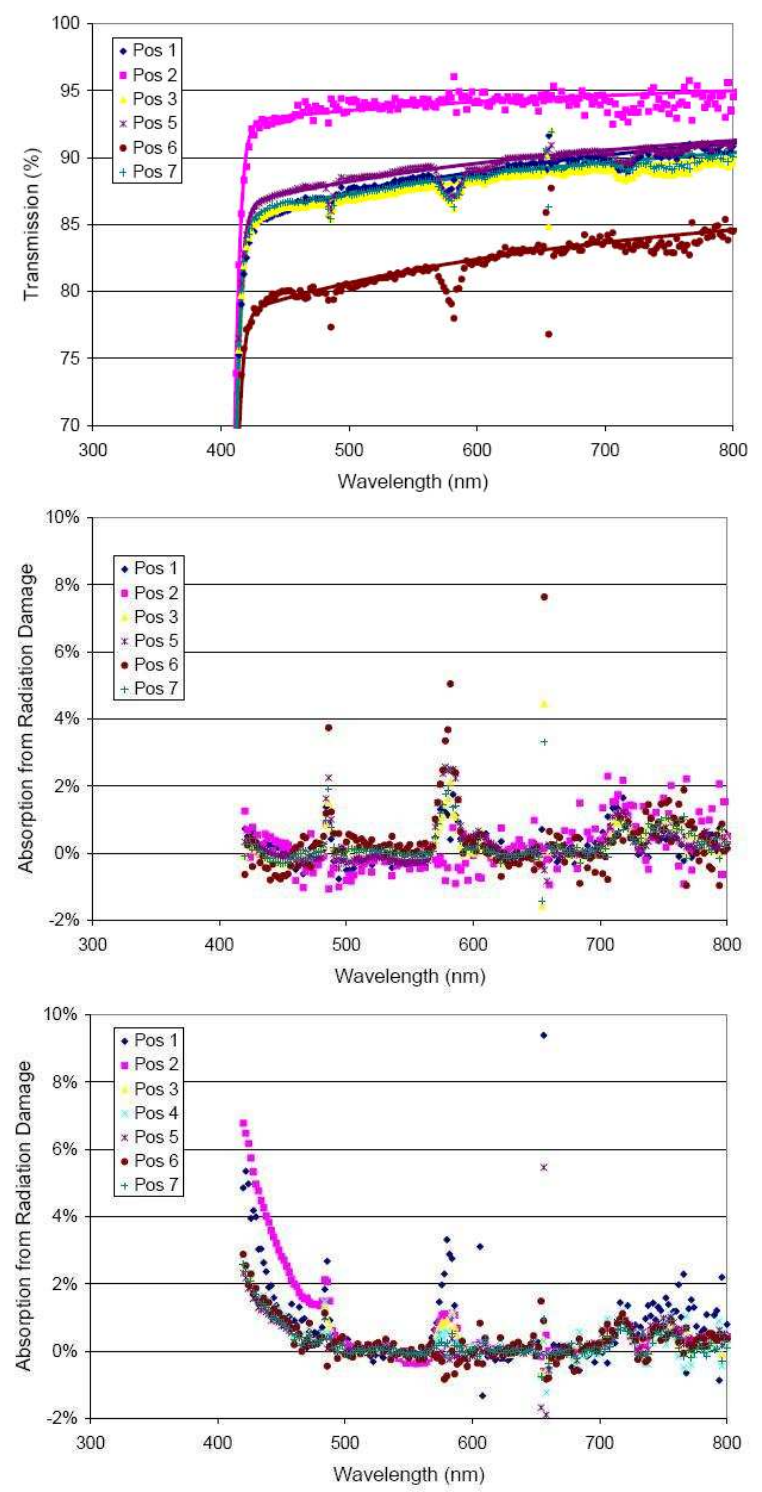

Fig. 3. The transmittance of a new scintillator as a function of wavelength (top) with the points showing the measured data and the solid line the fitted transmission function. The absorption for a new scintillator (middle) shows the transmission measurements are well modeled by the fit function aside from absorption peaks at discrete wavelengths. The absorption of an irradiated scintillator (bottom) shows evidence for radiation-induced absorption at the shorter wavelengths. Each figure inclues the results of seven different positions, labeled as Pos 1 to Pos 7 in the figure.

will be attenuated at shorter wave lengths, and thus exclude the wavelength region below $500 \mathrm{~nm}$ in the fits. Additionally, the following regions are also excluded because of absorption peaks: $570 \mathrm{~nm}<\lambda<590 \mathrm{~nm}, 650 \mathrm{~nm}<\lambda<660 \mathrm{~nm}$. To quantify the magnitude of the absorption $A$, we take a ratio of the measured transmission $T_{\text {meas }}$ and the fitted function $T_{f i t}$ as $A=1-\frac{T_{\text {meas }}}{T_{\text {fit }}}$. The results are shown in Fig. 3 .

The irradiated scintillator shows increased absorption due to radiation damage, especially at small radius where the radiation dose is expected to be highest. The scintillator light output, weighted by the photocathode quantum efficiency, peaks at 


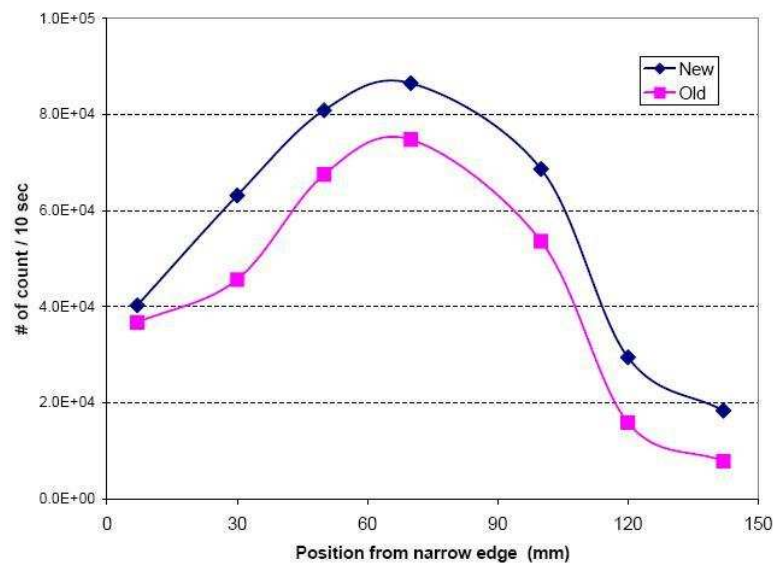

Fig. 4. The number of counts in recorded in $10 \mathrm{sec}$ for $\beta$-rays from a ${ }^{90} \mathrm{Sr}$ source as function of position in the scintillator. The pink points show the counting rate for an irradiated scintillator and the dark blue points show the rate for new scintillator using the same PMT.

$\sim 430 \mathrm{~nm}$, where we see $\sim 5 \%$ absorption in the $1.6 \mathrm{~cm}$ counter thickness. Because the typical photon path length for charged particles hitting the inner edge of the scintillator is $9 \mathrm{~cm}$, we expect the light output at small radii to be reduced by a factor of $0.95^{(9 / 1.6)}=0.75$ due to radiation damage.

\section{Counting RATE FOR A ${ }^{90} \mathrm{Sr} \beta$ SOURCE}

The counting rate from a $\beta$ source was measured for each counter. This measurement has two advantages over other measurements. First, it is a relatively quick measurement that we were able to perform on all 48 counters, allowing us to check for an azimuthal dependence and make a comparison between the counters mounted on the north and south ends of $D \emptyset$. Second, it provides a direct comparison between irradiated and new scintillators because we took data for both scintillators with same PMT and HV setting. The measurement process is as follows:

1) Setting HV: Equalizing the PMT gain. The number of photoelectrons $\left(N_{p e}\right)$ can be measured by using a charge distribution with irradiating LED pulse light. By assuming that LED light has Poisson distribution, $N_{p e}$ is calculated from mean $(\mu)$ and RMS $(\sigma)$ of charge distribution as $N_{p e}=(\mu / \sigma)^{2}$. By taking data with several points in HV, HV with the PMT gain of $5 \times 10^{6}$ is determined.

2) Setting threshold: The analog PMT signal was discriminated using a Lecroy 621BL NIM discriminator with a fixed threshold of $30 \mathrm{mV}$.

3) Measure counting rate: Set the ${ }^{90} \mathrm{Sr} \beta$ source, which had an initial activity of $1000 \mathrm{mCi}$, a distance of $7 \mathrm{~mm}$ from the narrow edge of the scintillator and measure the number of counts in a $10 \mathrm{sec}$ period using a visual scaler.

4) Change position and repeat. In total, we took data at seven different positions: $7 \mathrm{~mm}, 30 \mathrm{~mm}, 50 \mathrm{~mm}, 70 \mathrm{~mm}$, $90 \mathrm{~mm}, 120 \mathrm{~mm}$, and $150 \mathrm{~mm}$ from the narrow edge of the scintillator along the center line of the scintillator.
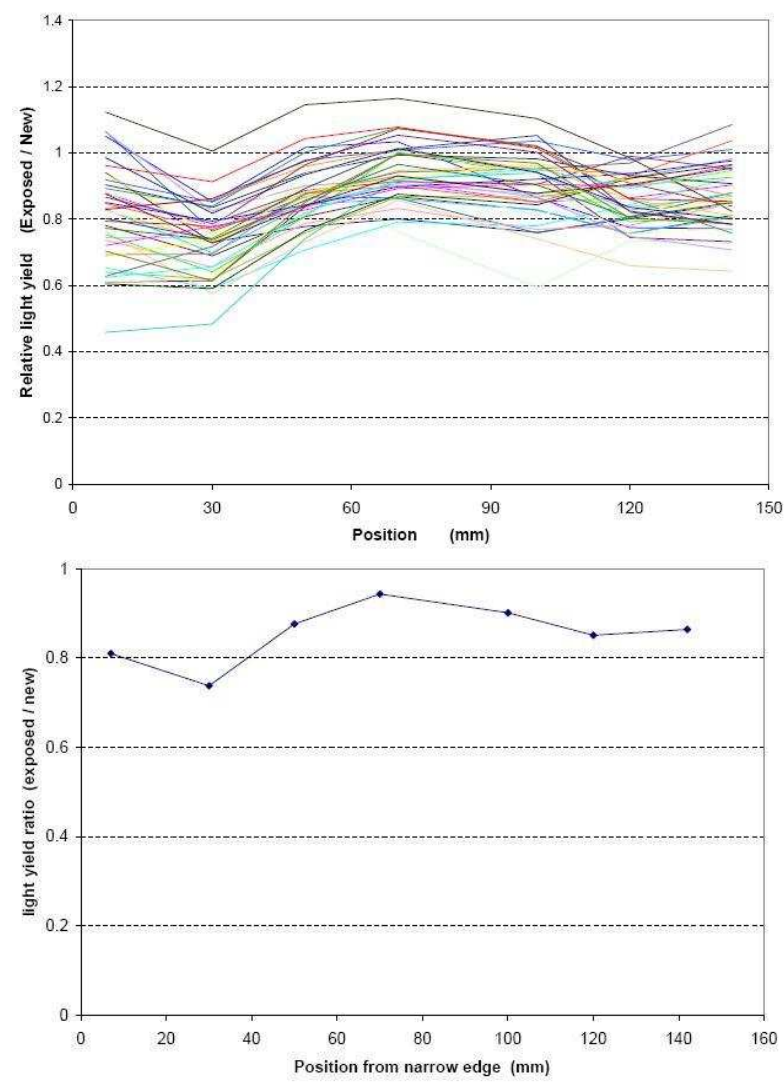

Fig. 5. The light yield of the irradiated scintillator relative to the new scintillator as function of position in the scintillator (top). There are 42 lines in this figure, with each line representing the results for one counter. The bottom figure shows the average of the light yield ratio for all counters vs position.

To obtain reliable results, we built a simple fixture to position the $\beta$ source on the LM counter. The positioning accuracy is better than $0.5 \mathrm{~mm}$ and $4 \mathrm{mrad}$. These measurements were performed in a black box with no reflective wrapping on the counters.

Typical results for irradiated and new scintillators using the same PMT are shown in Fig. 4. The count rate of irradiated scintillator is lower than for the new scintillator at all positions measured.

The challenge for this measurement is converting the source counting rate to light yield from because of the steeply falling spectrum of energy deposits. To obtain the light yield, we assume that an $\mathrm{x} \%$ reduction in light yield from radiation damage gives the same counting rate as an $\mathrm{x} \%$ reduction in the PMT gain. We can easily change the PMT gain by changing the PMT HV, and have measured that the PMT gain is proportional to $\mathrm{HV}^{7.1}$. Thus, by measuring the source count rate as a function of PMT HV, we can determine the count rate as a function of relative PMT gain, which we assume is equivalent to measuring the count rate as a function of relative light yield. We estimate this procedure introduces a $3 \%$ error in the relative light yield. After applying this procedure, we get the light yield of the irradiated scintillator relative to the new scintillator as function of position in the scintillator, as shown in Fig. 5(top). 


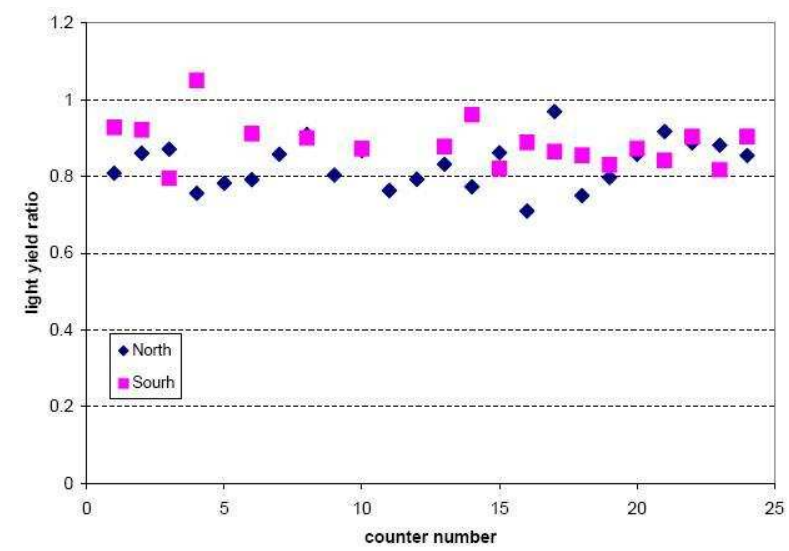

Fig. 6. The counter-to-counter variation in the light yield ratio. Each point shows the mean value of the light yield ratio averaged over the different positions. The dark blue points show the results for the 24 north counters and pink points show the results for the 24 south counters.

There is substantial counter to counter variation, but all counters show the same general trend.

Figure 5(bottom) shows the average light yield ratio for the 42 counter where the same PMT was used on the new counter. Irradiated counters are observed to a light yield that is $74-79 \%$ of the new counter at small radius. This result is consistent with the estimated light transmittance of $75 \%$ from the spectrophotometer measurements. We also observe a $\sim 10 \%$ light loss near the PMT, which is to be compared with our expectation of $\sim 5 \%$ from the transmittance measurements. There is no $\phi$ dependence in radiation damage as shown in Fig. 6. Some discrepancy between north side and south side can be seen. The average light yield ratio for the north side is $83 \%$, while it is $88 \%$ for the south side, whereas the radiation doses are expected to be almost identical for the north and south counters. It is possible that this discrepancy is due to annealing of the radiation damage through exposure to oxygen after they were removed from the detector. Almost all the north counters were measured in the third week after dismounting the LM from DØÖther hand, the south counters were measured on the fifth week. This time scale is not far from the speed of oxygen diffusion in the scintillator substrate, which has been measured to be $10^{-5} \mathrm{~mm} / \mathrm{s}$ [1].

\section{LIGHT YIELD FOR COSMIC RAYS}

The response of the LM counters to minimum ionizing particles has been measured using cosmic rays. To measure the light yield as function of hit position, we use two scintillation TOF counters as both trigger counters and as a tracking device. The TOF counters have PMTs on both ends, so the cosmic ray hit position in each TOF counter can be determined from the difference in time for the hits on the two ends using the light propagation speed in the scintillator. The cosmic ray is assumed to follow a straight-line trajectory between the hit positions in the two TOF counters.

The experimental setup for this measurement is shown in Fig. 7. We LM counter is placed in the middle between the

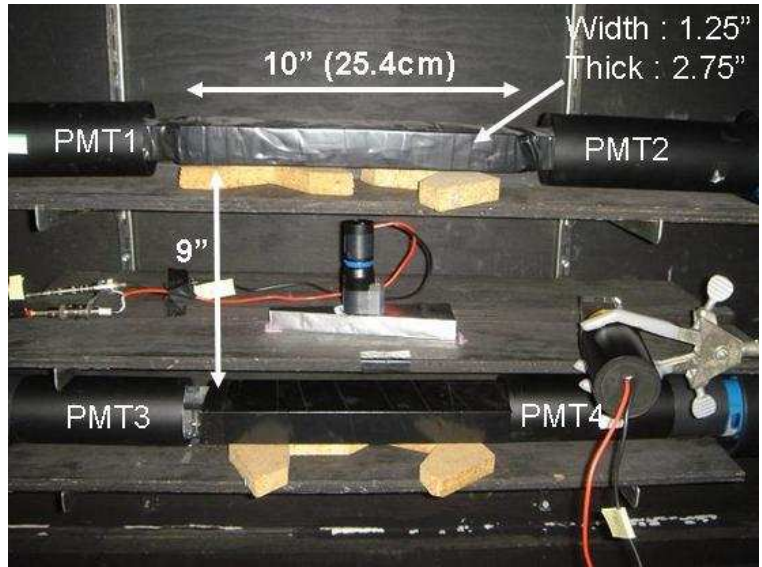

Fig. 7. Setup for the cosmic ray test stand.

two TOF counters. Each TOF counter consists of $3.2 \mathrm{~cm}$ thick Bicron BC408 scintillator bars that are $25.4 \mathrm{~cm}$ long and 7.0 $\mathrm{cm}$ wide, with Phillips XP2020 PMTs on each end. The thick scintillator gives rise to a large number of photoelectrons, giving rise to excellent timing resolution and a position resolution estimated to be $\sim 1 \mathrm{~cm}$. A trigger signal is generated by requiring a coincidence of the four TOF counter PMTs. The trigger timing is determined by PMT1 (upper left PMT of Fig. 7). To measure both charge and time information, each PMT output is divided in two by using a current divider. One output is sent to a NIM discriminator and the other to a CAMAC ADC module (Lecroy 2249W, resolution: 25pC/ADC count). The logic outputs from the discriminator are sent to a CAMAC TDC module (Lecroy 2228A, $50 \mathrm{ps} / \mathrm{TDC}$ count) and a NIM coincidence module that generates the trigger signal.

In this measurement, we measured 3 irradiated and 3 new counters using different PMTs whose gain was normalized to be the same using the method described in the previous section. The TOF counter PMT gains were equalized by adjusting the $\mathrm{HV}$ to yield a MIP peak at $\sim 100 \mathrm{pC}$.

To extract the hit position of the cosmic ray with good accuracy, we need to calibrate the TOF counters and correct for the time-walk effect. The fitting parameters and assumptions are listed below.

1) The propagation time ( $T^{i}, i$ is PMT number) in the scintillator is proportional to the distance from the hit position $\left(L_{h i t}^{i}\right)$ to the PMT as $T^{i}=L_{\text {hit }}^{i} / V_{\text {scint }}$, where $V_{\text {scint }}$ is the effective light propagation speed of 15 ( $\mathrm{cm} / \mathrm{ns})$.

2) The cosmic ray is assumed to travel with $\beta=1$.

3) The measured time $\left(T_{\text {meas }}\right)$ includes the time-walk effect. Pulses having low amplitude are delayed as $T_{\text {meas }}=$ $K / \sqrt{A D C}+T_{0}$ where $K$ and $T_{0}$ are free parameters in the fit.

4) The observed light yield in the LM counter has a position dependence due to the attenuation of light in the scintillator.

5) The TOF counters are set parallel with a separation of 

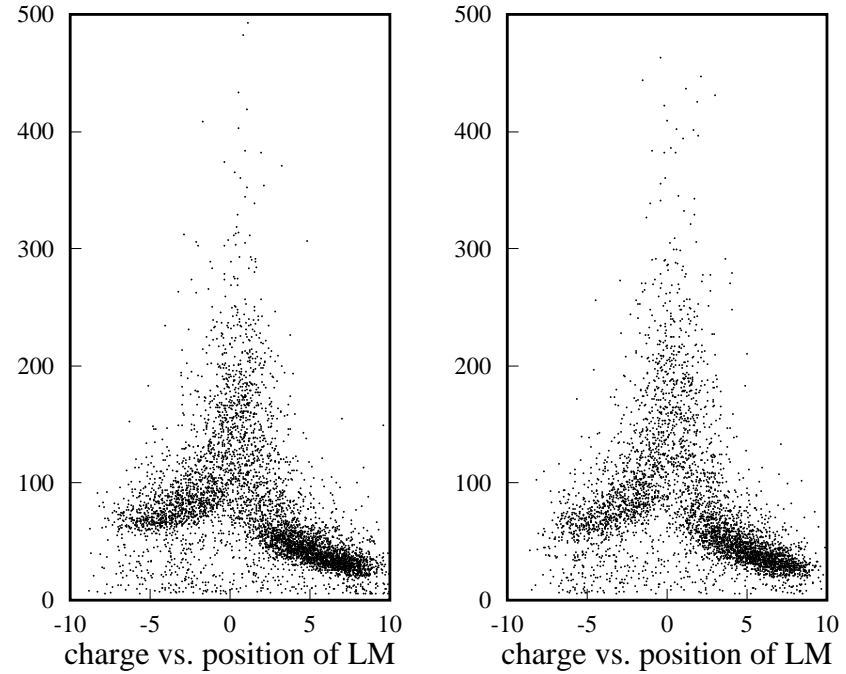

Fig. 8. Scatter plot of the measured charge vs hit position in the LM counter for new scintillator (left) and irradiated scintillator (right).

\section{$22.9 \mathrm{~cm}$.}

6) The cosmic ray comes from sky not ground.

In total, eight free parameters are determined by fitting.

Figure 8 shows scatter plots of charge vs position in the LM counter for both new (left) and irradiated (right) counters. The shape can be explained by the non-uniform light collection efficiency in the counters. The charge collected near the position $L \sim 0$, which is directly under the PMT, is greater than other hit positions due to having a very high light collection efficiency for hits directly under the PMT. The light yield at the narrow edge $(L \sim-6 \mathrm{~cm})$ is higher than at the wide edge $(L \sim+8$ $\mathrm{cm}$ ) because some photons traveling from the wide end towards the PMT will be reflected back by the side walls. The observed shape in Fig. 8 is well reproduce by a ray-tracing MC.

Figure 9(top) shows the average charge vs position for each of the three irradiated and new scintillators. The points in the figure is the mean value of each divided position. The light yield of irradiated counters is systematically lower than for the new counters. Averaging the measurements for the 3 new and 3 irradiated counters, we observe that the light yield near the narrow edge of the irradiated scintillator is $\sim 15 \%$ lower than for new scintillator, as shown in Fig. 9(bottom).

\section{CONCLUSION}

Four independent measurements have been performed to evaluate radiation damage in the LM scintillator during Run IIa. We observed a modest loss of light yield in region closest to beam pipe. Since the degradation in light yield from the transmittance measurement is consistent with both $\beta$ source and cosmic ray measurements, we conclude that the main source of degradation in light yield is due to increased light attenuation in the scintillator substrate rather than radiation damage to the scintillation mechanism. Based on these measurement, we replaced all LM scintillator during the spring 2006 TeVatron shutdown.
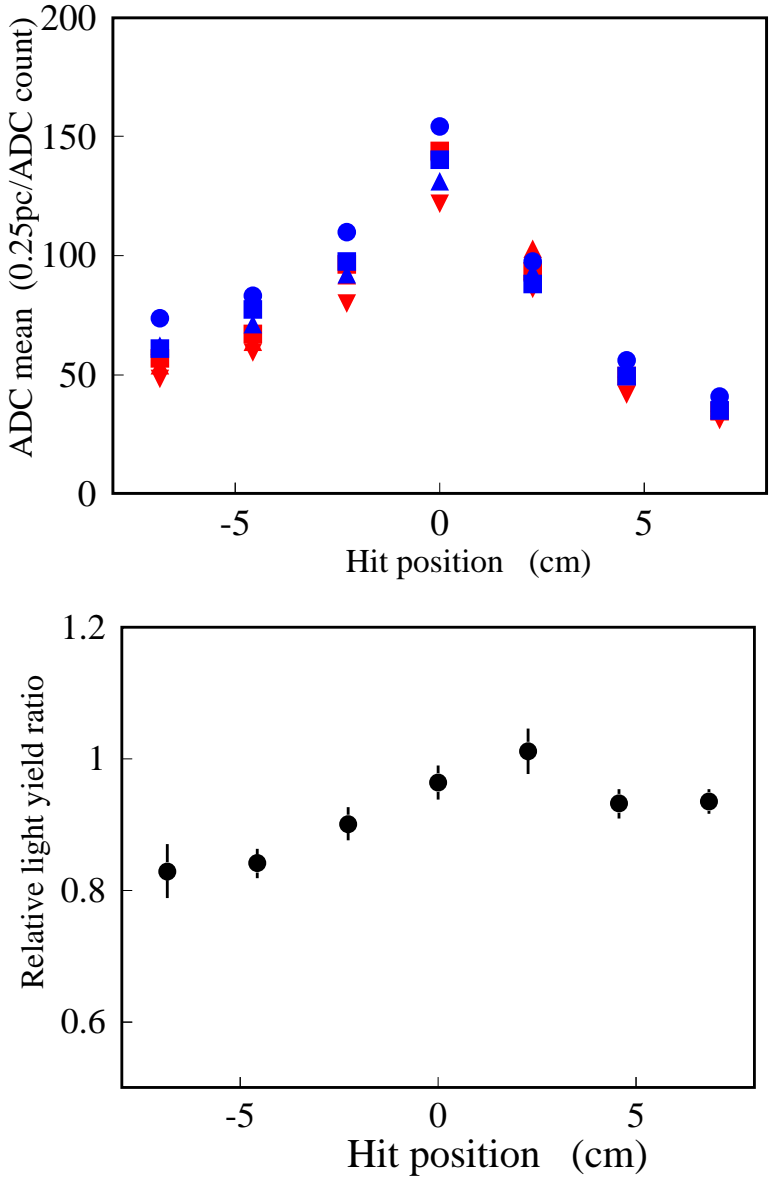

Fig. 9. top: The mean of measured charge vs hit position. Blue symbols show the result of three new scintillators, red symbols shows one of three exposed scintillator. bottom: The ratio of measured charge between new and exposed scintillators as function of hit position in the scintillator.

One source of uncertainty in these measurements is the effect of possible annealing of the radiation damage on the LM counters after they were removed from the detector. For most of the radiation dose accumulated by the scintillators, the enclosure containing the LM counters was connected to a nitrogen gas purge designed to minimize photomultiplier damage from ambient $\mathrm{He}$ in the collision hall. At the end of Run IIa, After dismounting the LM from the D $\varnothing$ detector, the LM counters were exposed in air. The transmittance measurements were performed after 3 days in air, while the $\beta$ source and cosmic ray tests were performed after being exposed to air for at least 3 weeks. It is not known if the oxygen content in the enclosures was high enough to allow the annealing process to occur, so it is possible that some annealing of the radiation damage occurred during the period these measurements were made.

\section{REFERENCES}

[1] A. Bross and A. Pla-Dalmau, Fermilab-Pub-91/74, March 1991. 